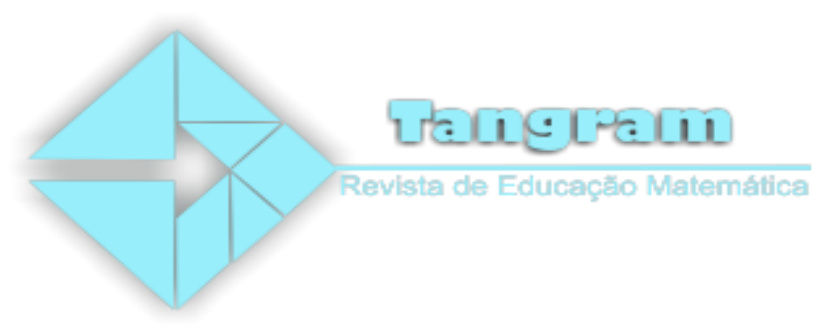

\title{
Estudio de la génesis instrumental del artefacto simbólico simetría axial
}

\section{Study of the instrumental genesis of symbolic artefact axial symmetry}

\author{
Daysi Julissa García-Cuéllar ${ }^{1}$ \\ Jesús Victoria Flores Salazar ${ }^{2}$
}

\begin{abstract}
Resumen: El presente artículo muestra una relectura de la tesis de maestría de la primera autora que trató del estudio del artefacto simbólico simetría axial con estudiantes de primer grado de secundaria (12 o 13 años de edad) de una escuela privada de Lima - Perú. Nuestro objetivo fue analizar el proceso de la Génesis Instrumental de dicho objeto matemático. Usamos como marco teórico al Enfoque Instrumental de Rabardel y como marco metodológico, aspectos de la Ingeniería didáctica de Artigue, centrándonos en el análisis a priori y a posteriori. Las acciones de los estudiantes permitieron identificar esquemas de utilización que construyeron y movilizaron, mientras interactuaban con el artefacto simbólico Simetría Axial. El uso del GeoGebra, en algunas actividades, favoreció la instrumentalización e instrumentación del objeto matemático abordado ya que permitió que los estudiantes validen sus conjeturas.
\end{abstract}

Palabras claves: Simetría Axial; Génesis Instrumental; Artefacto Simbólico; Ingeniería Didáctica.

Abstract: The present article shows a rereading of the master's thesis of the first author that dealt with the study of the symbolic artifact axial symmetry with first grade students of secondary (12 or 13 years old) of a private school of Lima - Peru. Our objective was to analyze the process of the Instrumental Genesis of said mathematical object. We use Rabardel's Instrumental Approach as a theoretical framework and, as a methodological framework, aspects of Artigue's didactic engineering, focusing on a priori and a posteriori analysis. The actions of the students allowed identifying utilization schemes that they built and mobilized, while interacting with the symbolic artifact axial symmetry. The use of

\footnotetext{
${ }^{1}$ Doctoranda en Educación matemática en PUC-SP. Instituto de Investigación sobre Enseñanza de las Matemáticas de la Pontificia Universidad Católica del Perú (IREM-PUCP). E-mail: garcia.daysi@pucp.pe

2 Doctora y post-Doctora en Educación Matemática en la Pontifica Universidade Católica de São Paulo. Profesora del Departamento de Ciencias/sección matemáticas y directora de la maestría en Enseñanza de las Matemáticas en la Pontificia Universidad Católica del Perú, PUCP. E-mail: jvflores@pucp.pe
}

Tangram - Revista de Educação Matemática, Dourados - MS - v.2 n. 3, pp. 28-48 (2019) 
GeoGebra, in some activities, favored the instrumentalization and instrumentation of the addressed mathematical object since it allowed the students to validate their conjectures.

Keywords: Axial Symmetry; Instrumental Genesis; Symbolic Artifact; Didactic Engineering.

Resumo: O presente artigo é uma releitura da dissertação da primeira autora que mostra um estudo do artefato simbólico Simetria axial com estudantes do primeiro ano do ensino secundário (12 ou 13 anos de idade) de uma escola particular de Lima - Peru. Nosso objetivo foi analisar o processo da Gênesis Instrumental desse objeto matemático. Utilizamos a Abordagem Instrumental de Rabardel como referencial teórico e como referencial metodológico, aspectos da Engenharia Didática de Artigue, com foco em análise a priori e a posteriori. As ações dos alunos permitiram identificar os esquemas de utilização que eles construíram e mobilizaram, interagindo com o artefato simbólico Simetria axial. O uso do GeoGebra, em algumas atividades, favoreceu a instrumentalização e instrumentação do objeto matemático abordado, porque permitiu aos estudantes validar suas conjecturas.

Palavras-chave: Simetria Axial; Gênese Instrumental; Artefato simbólico; Engenharia Didática.

\section{Introducción}

El objeto matemático simetría se encuentra en el currículo peruano de los años 2009 y 2017 (actual), en los ciclos V $\left(5^{\circ}\right.$ y $6^{\circ}$ grado de Educación Primaria) y VI $\left(1^{\circ}\right.$ y $2^{\circ}$ grado de Educación Secundaria). A pesar de estar presente en dichos documentos, la simetría axial y de manera general, la Geometría está siendo desarrollada de manera superficial por el poco tiempo que se le dedica en las aulas y/o tiene carencias en su enseñanza, tal como lo menciona el Ministerio de educación del Perú (MINEDU),

Se ha observado que los docentes desarrollan en menor tiempo y con menor profundidad las capacidades referidas a geometría. Si consideramos que las experiencias de aprendizaje no se circunscriben únicamente al último grado que se está cursando, sino que son la acumulación integradora de las experiencias a lo largo de toda la escolaridad, el que no se trabaje la geometría con el tiempo y la profundidad requeridas desde los grados anteriores puede ser una de las causas que influye negativamente en el aprendizaje de los estudiantes en este eje. (PERÚ, 2005, p.98).

En los resultados de la Evaluación Nacional del año 2004, realizada por la Oficina de Medición de la Calidad de los aprendizajes (UMC) del Ministerio de Educación del Perú, se puso de manifiesto que solo el 2,9\% de los estudiantes que estaban finalizando el quinto grado de secundaria, último año de la Educación Básica Regular del sistema educativo peruano, se encontraban en el nivel de suficiente en Geometría y Medición, que es el nivel 
esperado para todos los estudiantes dicho grado. Esto se da por la prioridad que se da al cálculo y no al desarrollo de capacidades fundamentales.

[...] Y, ante la ausencia de un enfoque pedagógico, la mayoría de profesores optó por trabajar asociando la matemática con la capacidad de calcular. En primaria, lo fundamental era el dominio de los cálculos aritméticos y, en secundaria, el cálculo algebraico, geométrico y trigonométrico. Este regreso a lo básico prevalece hasta hoy en día en la práctica docente, hecho que puede comprobarse por la existencia de estudios que señalan que casi el $85 \%$ de los ejercicios resueltos por los estudiantes en sus cuadernos de trabajo y de clase se centran en la aplicación de algoritmos convencionales (PERU, 2005, p.18).

Los resultados de la Evaluación Censal de Estudiantes (ECE) ${ }^{3}$, aplicada a 490637 alumnos de $2^{\circ}$ grado de Educación Secundaria matriculados oficialmente en el sistema educativo peruano en el año 2015, indican que hubo una pequeña mejora en los resultados de los aprendizajes de los estudiantes. Sin embargo, en el 2016, que fue la última aplicación de una ECE, solo el 11,5\% de los estudiantes del grado mencionado, se encontraron en el nivel satisfactorio, esto se puede apreciar en la figura 1.

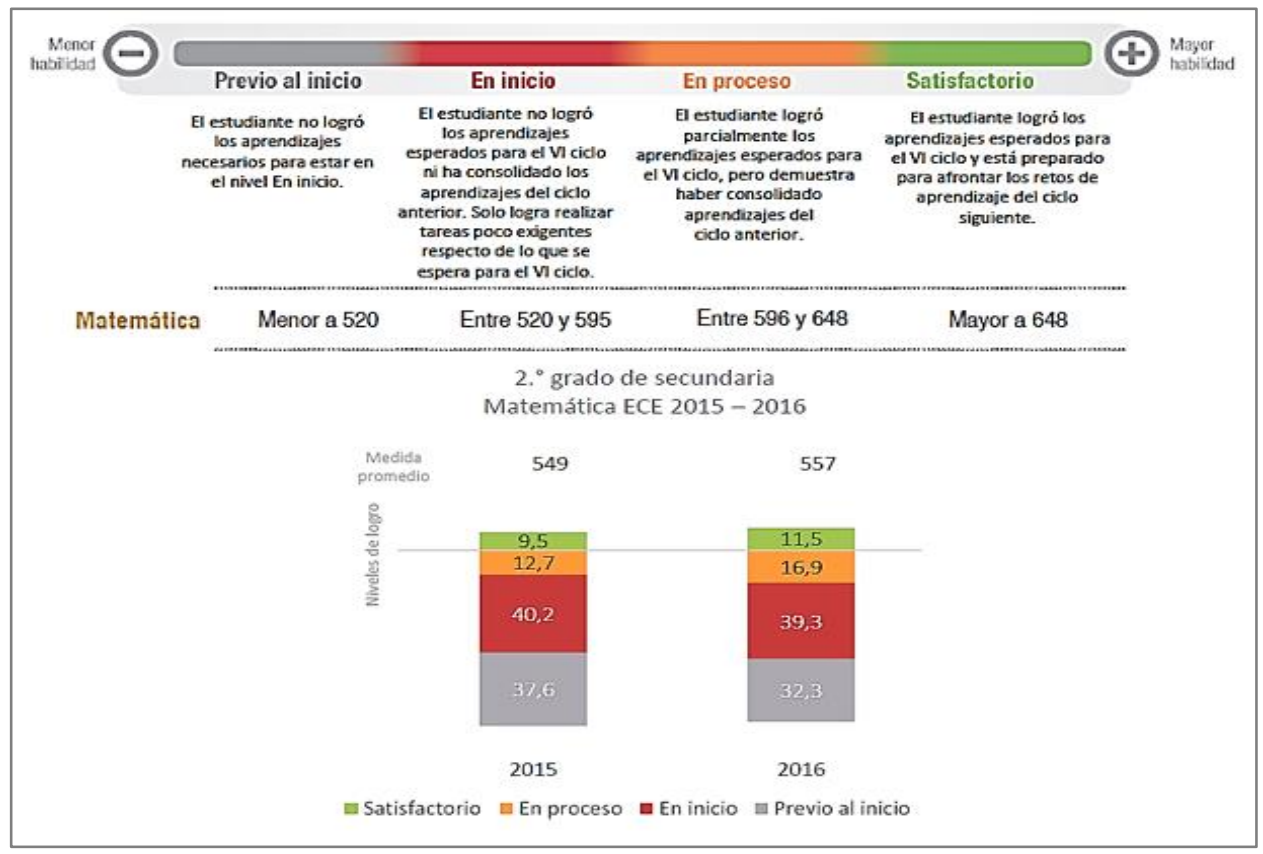

Figura 1. Resultados de la Evaluación Censal de Estudiantes 2015-2016. (PERU, 2016)

\footnotetext{
${ }^{3}$ La Evaluación Censal de Estudiantes (ECE) es una evaluación estandarizada que realiza el Ministerio de Educación del Perú, a través de la Oficina de Medición de la Calidad de los Aprendizajes (UMC), para saber qué y cuánto están aprendiendo los estudiantes de las escuelas públicas y privadas del país.
}

8Tangram - Revista de Educação Matemática, Dourados - MS - v.2 n. 3, pp. 28-48 (2019) 


\section{Estudio de la génesis instrumental del artefacto simbólico simetría axial}

De lo anterior, podemos apreciar que los estudiantes de secundaria del sistema educativo peruano todavía tienen dificultades en el aprendizaje de la matemática y en especial de la Geometría. Desde nuestra experiencia profesional, hemos notado que en los años de escolaridad hay temas como las transformaciones geométricas en el plano, la geometría analítica, entre otros; que no se enseñan a pesar que están en curriculo, pensamos que esto sucede por falta de tiempo o porque se profundiza en otros temas.

Una de las transformaciones geométricas en el plano es la simetría, ésta tiene varias aplicaciones como, por ejemplo: En la música clásica, donde existen composiciones en las que podemos encontrar distribuciones de las notas generadas mediante simetría. Algunas composiciones como: El Preludio de Johann Sebastian Bach, la Sonata en G mayor de Domenico Scarlatti, Lotosblume de Robert Schumann, o Die Meiestersinger de Richard Wagner. En la arqueología, encontramos simetría en las diferentes manifestaciones culturales de nuestros antepasados, un ejemplo es el lanzón monolítico que se encuentra en el templo de Chavín de Huántar en Perú. En el arte, como la simetría percibida en las distintas obras artísticas del holandés M. C. Escher. En la biología, como la simetría presente en las hojas, en las flores y en animales. También la encontramos en la física, como la simetría que se puede observar en los gráficos de los movimientos armónicos simples.

[...] la simetría impregna toda la ciencia y ocupa un lugar prominente en la química, la biología, la fisiología y la astronomía. La simetría abarca desde el mundo íntimo de la estructura de la materia hasta el inmenso dominio cósmico, pasando por el universo abstracto de las matemáticas. (Lederman, L, 1996, p.229).

Según García-Cuéllar (2014), la simetría es un contenido matemático importante debido a que tiene implicancia en el aprendizaje de otros contenidos o conocimientos que los estudiantes van a aprender en los últimos años de educación secundaria y/o primeros ciclos universitarios como, por ejemplo: el aprendizaje de la parábola, elipse e hipérbola, así como también en el aprendizaje de las funciones inversas y funciones pares. La simetría proporciona oportunidades para que los estudiantes visualicen la geometría en el mundo desde el arte, la naturaleza, las construcciones arquitectónicas, como ya lo hemos mencionado anteriormente, pero lo más importante es que permite la exploración de regularidades y/o patrones que representan la esencia del conocimiento matemático.

Existen diversas investigaciones en Educación Matemática que tratan sobre los problemas presentes en la enseñanza y aprendizaje de este objeto matemático, como la de 
Ferreira (2005) que realizó un estudio de las isometrías dando énfasis a la simetría, donde hace un análisis en los documentos curriculares como la Propuesta Curricular Nacional del Brasil; también analiza las actividades de algunos textos escolares presentan nociones de isometrías y con ello el estudio de la simetría. La investigadora manifiesta que no hay un estudio profundo de la noción de simetría en los textos escolares, por ello, menciona que la simetría es uno de los contenidos que no se profundiza en los libros de textos escolares del nivel medio en Brasil y que es necesario realizar más estudios. Este problema no es diferente en el Perú, en los libros didácticos, el objeto matemático Simetría axial es presentado como algo acabado que solo necesita ser aplicado por los estudiantes en las actividades, como una repetición de modelos dados como ejercicios resueltos o ejemplos.

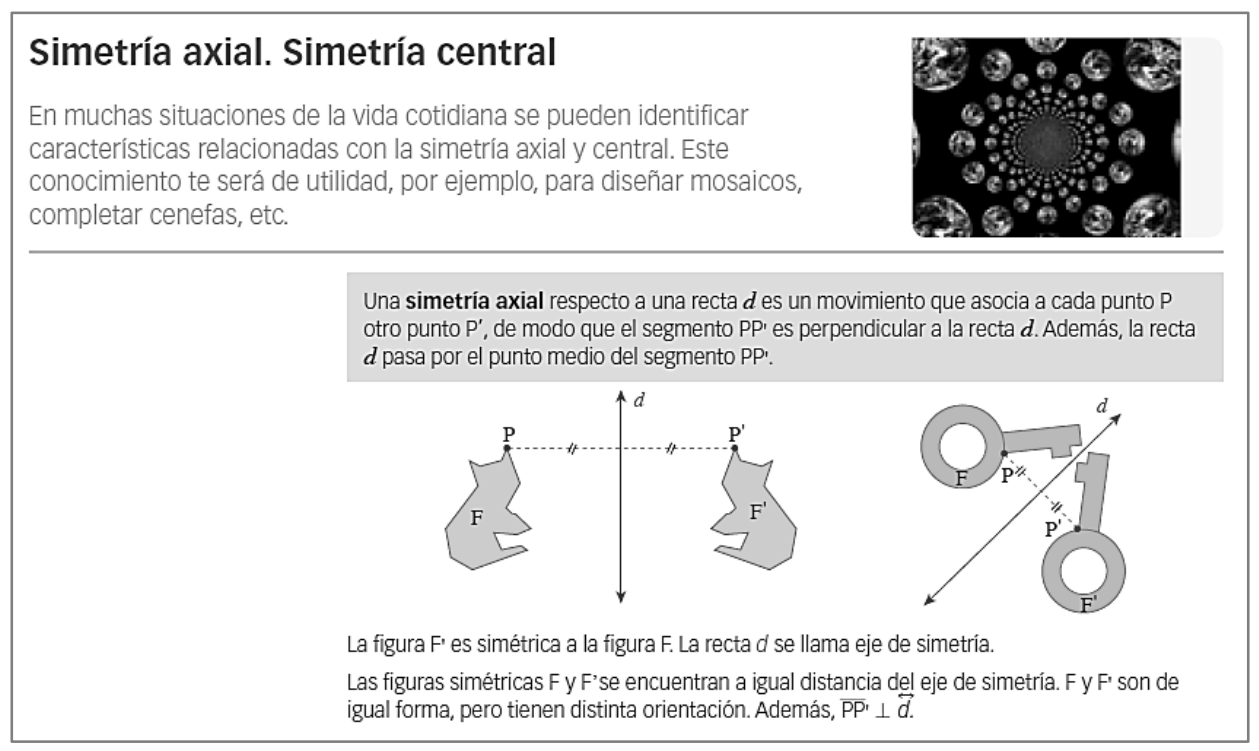

Figura 2. Libro matemática 1. (Santillana, 2018, p.42).

En la figura 2, se muestra cómo es introducido el objeto Simetría axial, en el libro de texto Matemática 1 del primer grado de Educación Secundaria. Se puede observar que el concepto de simetría axial y sus propiedades es presentado a los estudiantes sin actividades que permitan deducir, analizar, verificar y descubrir este concepto. Posteriormente a esta introducción, se presentan dos ejemplos (ejercicios resueltos) donde prima el análisis gráfico, es decir, el cómo realizar el simétrico de una figura o de un polígono, o cómo determinar el eje de simetría de una figura. Finalmente, las actividades propuestas son del mismo tipo que los ejemplos, es decir, el estudiante solo repite los modelos de solución dados.

8Tangram - Revista de Educação Matemática, Dourados - MS - v.2 n. 3, pp. 28-48 (2019) 


\section{Estudio de la génesis instrumental del artefacto simbólico simetría axial}

Otras investigaciones como las de Jaime (1993), Ferreira (2005), Ulian (2008) y Lima (2006) muestran que existe una problemática en la enseñanza y en el aprendizaje de la noción de simetría, como el de identificación de figuras simétricas y problemas de construcción de figuras simétricas. Investigaciones como las de Salazar (2009) y Bettini (2017) nos brindan información sobre el papel que tiene el uso de la tecnología en el aprendizaje de la geometría, pues, por medio de los softwares, específicamente de los softwares de geometría dinámica, los estudiantes pueden hacer conjeturas, verificar hipótesis, etc.

En la misma línea de pensamiento, la investigación de Gallegos y Peña (2012) muestra que en las últimas décadas, muchas pesquisas en el área de Educación Matemáticas se centran en el uso de estas herramientas para la enseñanza y el aprendizaje de contenidos matemáticos. Precisamente, ellos afirman que:

La integración y utilización de las tecnologías en el proceso educativo de matemáticas es un asunto que viene ocupando el trabajo de los investigadores en educación matemática. Las investigaciones tratan de determinar los posibles beneficios que la utilización de las tecnologías conlleva, así como diversas metodologías y entornos interactivos multimedia de aprendizaje que produzcan mejoras en los procesos de enseñanza y aprendizaje (Gallegos y Peña, 2012, p.11).

Además de esta investigación, el Ministerio de Educación del Perú en sus Orientaciones para el trabajo pedagógico (Perú, 2011) también menciona la pertinencia del uso de la tecnología, especialmente, para la geometría,

[...]La tecnología desempeña también un papel importante en la enseñanza y el aprendizaje de la geometría. Herramientas como un programa informático de "Geometría dinámica", capacitan para modelizar una gran variedad de figuras de dos dimensiones y para tener una experiencia interactiva con ellas. La visualización y el razonamiento espacial se enriquecen mediante la interacción con animaciones de ordenador y en otros contextos tecnológicos (p. 30).

Por todo lo mencionado anteriormente, vimos pertinente hacer un estudio de la simetría axial, que ayude al estudiante a deducir las propiedades de la simetría y sus aplicaciones. Como nuestro interés es analizar el proceso de la Génesis Instrumental del artefacto simbólico simetría axial, cuando resuelve actividades usando GeoGebra o lápiz y papel, usamos como marco teórico al Enfoque instrumental de Rabardel (1995) y como 


\section{Estudio de la génesis instrumental del artefacto simbólico simetría axial}

método de investigación algunos aspectos de la Ingeniería didáctica de Artigue (1995), que vamos a explicar a continuación.

\section{Aspectos del enfoque instrumental}

El Enfoque Instrumental aborda la dimensión tecnológica de la educación matemática, articulando los aspectos importantes de la integración tecnológica en el proceso de enseñanza y aprendizaje de las matemáticas.

Salazar (2009), en su investigación, presenta las nociones claves de este Enfoque son las siguientes: Esquema: Es una organización invariante de la conducta del sujeto para una clase determinada de situaciones. Artefacto: Es un objeto material o simbólico, destinado a dar sustento a la actividad del sujeto en la ejecución de un cierto tipo de tarea. Instrumento: Es lo que un sujeto construye a partir del artefacto; es entonces una entidad mixta que contiene a la vez un artefacto, material o no, y esquemas de utilización construidos por el sujeto durante su interacción.

Así, de acuerdo a Rabardel (1995), el Enfoque Instrumental estudia la diferencia que existe entre el artefacto, instrumento y los procesos que desenvuelven la transformación progresiva del artefacto en instrumento, transformación que denominó como proceso Génesis Instrumental. El autor considera tres polos importantes en la Génesis instrumental, estos son: el sujeto, que puede ser un usuario, operario, trabajador o agente; el instrumento, que se refiere de la herramienta, máquinas, sistemas, utensilio, etc.; y el objeto, al cual va dirigida la acción con ayuda del instrumento, este puede ser la materia prima, objeto de la actividad o trabajo.

El investigador sostiene que el instrumento no existe en sí, sino que es el resultado de asociar el artefacto a la acción del sujeto, como medio para la misma. En nuestro caso, el artefacto simbólico es la simetría axial. El autor señala que el artefacto pasará al estado de instrumento, cuando el sujeto le asigne los esquemas de utilización correspondientes.

En cuanto a la génesis instrumental, esta consta de dos dimensiones: La instrumentalización y la instrumentación.

Los procesos de instrumentalización están dirigidos hacia el artefacto: selección, agrupación, producción e institución de funciones, usos desviados, atribuciones de propiedades, transformaciones del artefacto, de su estructura, de su funcionamiento, etc. [...] los procesos de Instrumentación están relacionados con el sujeto: con la emergencia y evolución de los esquemas sociales de utilización y de acción instrumentada: su constitución, su evolución por

8Tangram - Revista de Educação Matemática, Dourados - MS - v.2 n. 3, pp. 28-48 (2019) 


\section{Estudio de la génesis instrumental del artefacto simbólico simetría axial}

acomodación, coordinación y asimilación recíproca, la asimilación de artefactos nuevos a los esquemas ya constituidos, etc. (Rabardel, 1995, p. 215).

Por lo anterior, las dos dimensiones de la Génesis Instrumental, dependen de su orientación:

La instrumentalización está dirigida hacia la parte artefactual del instrumento, consta del enriquecimiento de las propiedades del artefacto por parte del sujeto. Es decir, es el resultado de la atribución de una función al artefacto por parte del sujeto.

La instrumentación está dirigida hacia el sujeto. Se refiere a la construcción de esquemas de uso por parte del sujeto, relativos a la ejecución de ciertas tareas. En este proceso se lleva a cabo la asimilación de nuevos artefactos a los esquemas y la acomodación de los esquemas para dar nuevos significados a los artefactos. En la figura 3, se puede observar las dos dimensiones del proceso de la Génesis Instrumental.

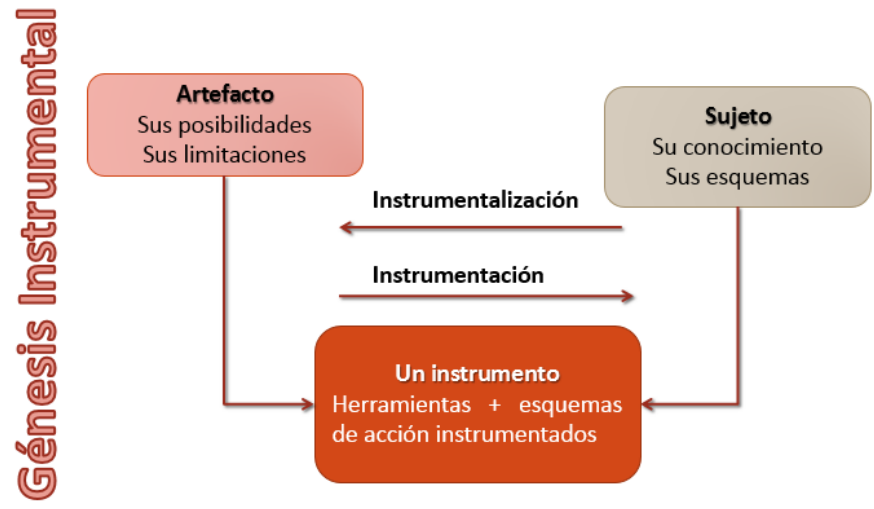

Figura 3. Proceso de la Génesis Instrumental. Adaptado de Trouche (2004).

Bellemain y Trouche (2016) sostiene que estas dos dimensiones de la Génesis Instrumental no son independientes una de la otra, sino que son entrelazadas. Pero, para distinguirlos en el análisis, se puede focalizar por un lado en el estudiante (¿En qué medida la integración de un nuevo artefacto modifica la forma de su actividad?), y por otro lado, en el artefacto (¿En qué medida este aporta al vestigio de la actividad del estudiante, de su poder creativo?).

Rabardel (2011), a partir de esta noción de esquema de Vergnaud ${ }^{4}$ (1996), define los esquemas de utilización como el conjunto estructurado de las características generalizables

\footnotetext{
${ }^{4}$ Para Vergnaud (1996), un esquema es una organización invariante de la actividad para una clase de situación dada. Está formado necesariamente por cuatro componentes: Un objetivo, sub-objetivo y anticipaciones. 8Tangram - Revista de Educação Matemática, Dourados - MS - v.2 n. 3, pp. $28-48$ (2019)
} 


\section{Estudio de la génesis instrumental del artefacto simbólico simetría axial}

de la acción que permiten repetir la misma acción o aplicarlas en nuevos contextos. Estos esquemas, a la vez, pueden ser clasificados en esquemas de uso (dirigidas a tareas secundarias), esquemas de acción instrumentada (dirigidas a la tarea principal o primaria) y esquemas de acción colectiva instrumentada (cuando el colectivo comparte el mismo instrumento o trabaja con la misma clase de instrumento, buscando alcanzar una meta en común). En nuestro estudio nos centraremos en los esquemas de uso y de acción instrumentada.

\section{Metodología}

Nuestra metodología es de corte cualitativo, en ese sentido, Denzin y Lincoln (1994) sostienen que la metodología cualitativa es multimetódica, naturalista e interpretativa. Es decir, que las investigadoras e investigadores cualitativos indagan en situaciones naturales, intentado dar sentido o interpretar los fenómenos en los términos del significado que las personas les otorgan. Asimismo, los investigadores cualitativos tienen más interés por el proceso que por los resultados o productos.

En cuanto a nuestro método de investigación, utilizaremos aspectos de la Ingeniería Didáctica de Artigue (1995). La noción de ingeniería didáctica surgió en la didáctica de las matemáticas a comienzos de los años ochenta. Se denominó con este término a una forma de trabajo didáctico equiparable con el trabajo del ingeniero, quien, para realizar un proyecto determinado, se basa en los conocimientos científicos de su dominio y acepta someterse a un control de tipo científico. Al igual que un ingeniero, la autora sostiene que el profesor concibe, realiza, observa y analiza secuencias de enseñanza para lograr el aprendizaje de un contenido matemático determinado por un grupo específico de estudiantes.

[...] el trabajo del ingeniero que, para realizar un proyecto preciso, se basa en los conocimientos científicos de su dominio y acepta someterse a un control de tipo científico. (Artigue, 1995, p.33).

Para Almouloud (2007), la Ingeniería Didáctica se apoya en un esquema experimental basado en la concepción, realización, observación y análisis de una secuencia de enseñanza, además de la validación, que es la comprobación o no de los supuestos asumidos en el estudio, mediante el análisis a priori y a posteriori. Además de eso, el método

Reglas de acción, formada de informaciones y control. Invariantes operatorias (reglas de acción y teoremas en acción). Posibilidades de inferencias en una situación.

8Tangram - Revista de Educação Matemática, Dourados - MS - v.2 n. 3, pp. 28-48 (2019) 
es caracterizado como experimental porque puede ser utilizada en investigaciones que estudian los procesos de enseñanza y aprendizaje de un objeto matemático.

La ingeniería didáctica tiene las siguientes fases: Análisis preliminar, en donde se realiza el análisis distinguiendo tres dimensiones: epistémico, cognitivo y didáctico. En nuestra investigación, en la dimensión epistémica desarrollamos un estudio históricoepistemológico de la simetría considerándola como una transformación en el plano, también presentamos una definición formal de la simetría. En la dimensión cognitiva, realizamos la revisión de investigaciones cuyo objeto de estudio es la simetría, para identificar la problemática en torno a la enseñanza y/o aprendizaje de este objeto matemático. En la dimensión didáctica se realizó el análisis de los libros didácticos del primer grado de educación secundaria. Concepción y análisis a priori: Se realizó el conjunto de supuestos sobre lo que harán los estudiantes. Experimentación: En esta fase predomina el acercamiento entre el docente investigador y la población de estudiantes sujetos de la investigación. También se aplican los instrumentos diseñados por el investigador y se lleva a cabo los registros de observación de la experiencia. Análisis a posteriori y validación: Según la Artigue (1995), en esta etapa, se realiza el análisis de los datos recolectados durante los diferentes momentos de la experiencia. En esta fase, analizamos los datos recolectados en los dos encuentros realizados con las estudiantes en el laboratorio de informática y los contrastaremos con el análisis a priori. En el presente artículo hacemos énfasis en las fases de análisis a priori y el análisis a posteriori de la Ingeniería didáctica.

\section{Experimentación y resultados}

La parte experimental se realizó con estudiantes de primer grado de Educación secundaria (12 o 13 años de edad), en una sala de informática en una escuela particular de Lima- Perú. La secuencia de actividades se realizó en dos encuentros donde se aplicaron tres tipos de actividades: la actividad $\mathrm{N}^{\circ} 0$, que tuvo la finalidad de familiarizar a los estudiantes con algunas herramientas del GeoGebra; la actividad $\mathrm{N}^{\circ} 1$, donde se centró en el desarrollo de esquemas de utilización de la simetría axial y, finalmente, proponemos la actividad $\mathrm{N}^{\circ} 2$, donde se buscó que los estudiantes pongan en acción sus esquemas de utilización sobre el artefacto simbólico de simetría axial. Esta organización se puede observar en la tabla 1 que se presenta a continuación. 
Estudio de la génesis instrumental del artefacto simbólico simetría axial

Tabla 1. Descripción de los encuentros de aplicación de las actividades

\begin{tabular}{|c|c|c|}
\hline Actividad & Encuentro & contenido \\
\hline${\text { Actividad } \mathrm{N}^{\circ} 0}^{\circ}$ & I & Introducción al GeoGebra \\
\hline Actividad $\mathrm{N}^{\circ} 1(\mathrm{~A}, \mathrm{~B}, \mathrm{C}, \mathrm{D}$ y E) & I y II & Simetría Axial \\
\hline Actividad $\mathrm{N}^{\circ} 2(\mathrm{~A}, \mathrm{~B}$ y C) & II & Aplicaciones \\
\hline
\end{tabular}

A continuación, presentamos el análisis a priori y a posteriori de las actividades 1C, 1D, 2A y 2C que se elaboró y se planteó en la investigación. Cabe señalar que las actividades 1A y 1B fueron tratados en un artículo anterior. Para el análisis a posteriori, en este artículo, utilizamos los procesos realizado por una estudiante que denominamos Marcia.

\section{Actividad 1 C}

Abre el archivo actividad1_C.ggb.Utilizando la herramienta punto medio, traza los puntos medios de los segmentos AA', BB', CC', DD', EE', FF', GG', HH' e II'. Con la herramienta recta, traza la recta que pasa por los puntos medios marcados anteriormente. Anota tus observaciones.

Traza el segmento AA' y mide un ángulo que se forma entre la intersección del segmento y la recta. ¿Cuánto mide el ángulo?

Traza BB' y mide un ángulo que se forma entre la intersección del segmento y la recta. ¿Cuánto mide el ángulo? ¿Qué puedes concluir con respectos a los ángulos que se forman entre la intersección de la recta y los segmentos trazados? Justifica

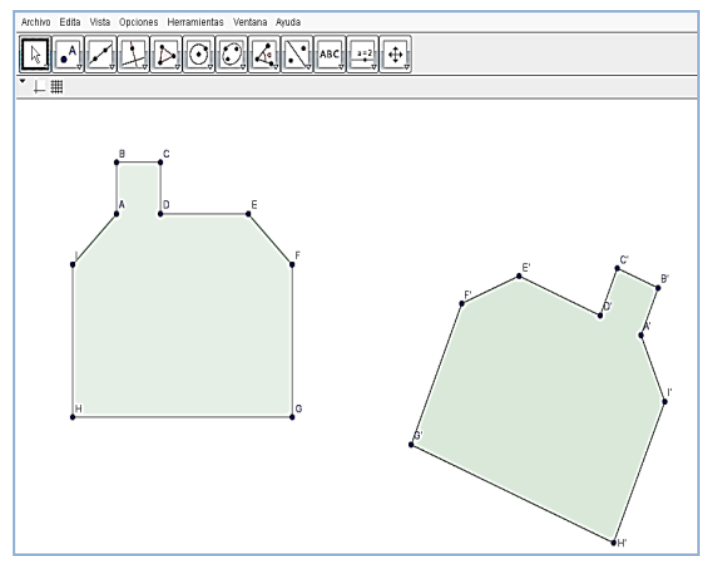

Figura 4. Enunciado de la Actividad 1C

A priori, la actividad tiene como objetivo trazar el eje de simetría no vertical, es decir, con inclinación y sin cuadrículas, dado que en la actividad 1A y 1B se trabajó con el eje de simetría vertical. Los estudiantes debían elegir la herramienta punto medio y traza los puntos medios de los segmentos AA', BB', CC', DD', EE', FF', GG', HH' e II'. Luego, traza la recta que pasa por los puntos medios marcados anteriormente. Finalmente, medir los ángulos que se forman entre la intersección de la recta y los segmentos trazados, observar que son ángulos rectos.

Para dar solución a la actividad, los posibles esquemas de uso de los estudiantes serían: recta, segmentos, punto medio, ángulos, perpendicularidad. Y el posible esquema de acción instrumentada serían eje de simetría como mediatriz de los segmentos AA', BB', CC', DD', EE', FF', GG', HH' e II'.

A posteriori, Marcia trazó los puntos medios de AA', BB', CC', DD', EE', FF', GG', HH' e II' y luego utilizando la herramienta recta que pasa por dos puntos, traza la recta que contiene a todos los puntos medios. También haciendo uso de la herramienta recta que pasa 8Tangram - Revista de Educação Matemática, Dourados - MS - v.2 n. 3, pp. 28-48 (2019) 
por dos puntos. Trazó las rectas que pasan por los puntos A y A' así como también la recta que pasa por B y B'. Posteriormente, medió los ángulos formados en la intersección de la recta $\mathrm{AA}^{\prime}, \mathrm{BB}$ ' con la recta que contiene los puntos medios, tal como se muestra en la figura 5.

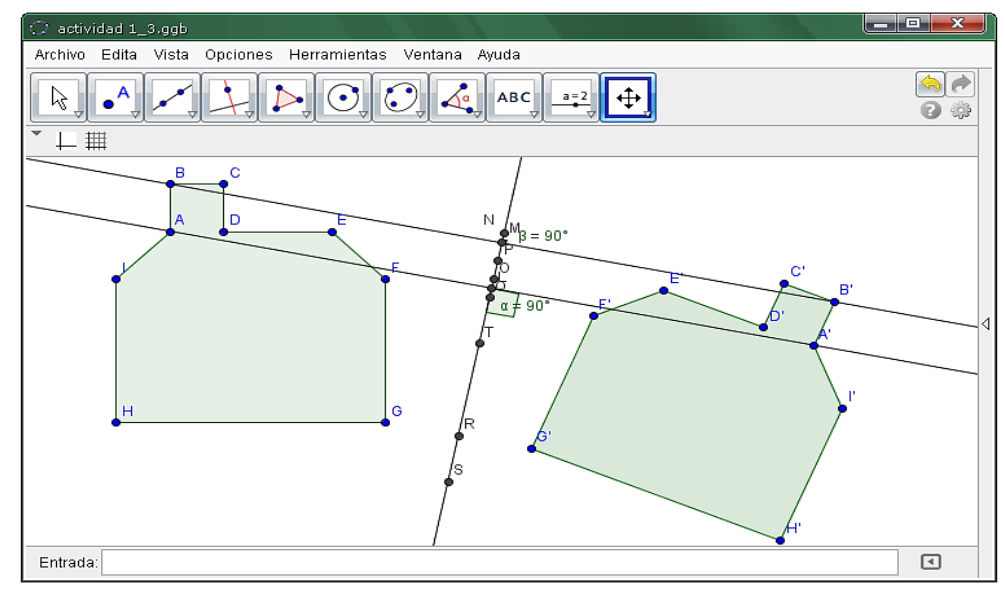

Figura 5. Solución de Marcia en la actividad 1C

Marcía logró identificar que la recta que trazó contiene a todos los puntos medios de ambos polígonos como lo menciona en la figura 6 (a). Asimismo, logró darse cuenta que en la intersección de los segmentos con la recta se forman ángulos rectos, tal como lo menciona en la figura 6 (b), que muestra un recorte de su ficha de trabajo.

Abre el archivo actividadl_3.ggb.Utilizando la herramienta punto medio, traza los puntos
medios de los segmentos AA', BB', CC', DD', EE', FF', GG', HH' e II'. Con la
herramienta recta, traza la recta que pasa por los puntos medios marcados anteriormente.
Anota tus observaciones.
rodos los puxtos se unen a krovês de una recta.

(a)

Traza el segmento $\mathrm{AA}^{\prime}$ y mide un ángulo que se forma entre la intersección del segmento
y la recta. ¿Cuánto mide el ángulo? $90^{\circ}$
un ángulo que se forma entre la intersección del segmento y la recta. ¿Cuánto mide el
ángulo? iqué puedes concluir con respectos a
los ángulos que se forman entre la intersección de la recta y los segmentos trazados?
justifica. que nunca varía su nedi da. y a traves de vina recta re
pue de oríginar ánguios.

(b)

Figura 6. Respuesta de Marcia en la actividad 1C

Con respecto a nuestro análisis a priori, Marcia logró los objetivos previstos para esta actividad. Las acciones de Marcia nos dan indicios de sus esquemas de uso como: ángulo, recta, punto medio y mediatriz. Por lo anterior, podríamos decir que generó el esquema de acción instrumentada eje de simetría como mediatriz. 


\section{Actividad $1 \mathrm{D}$}

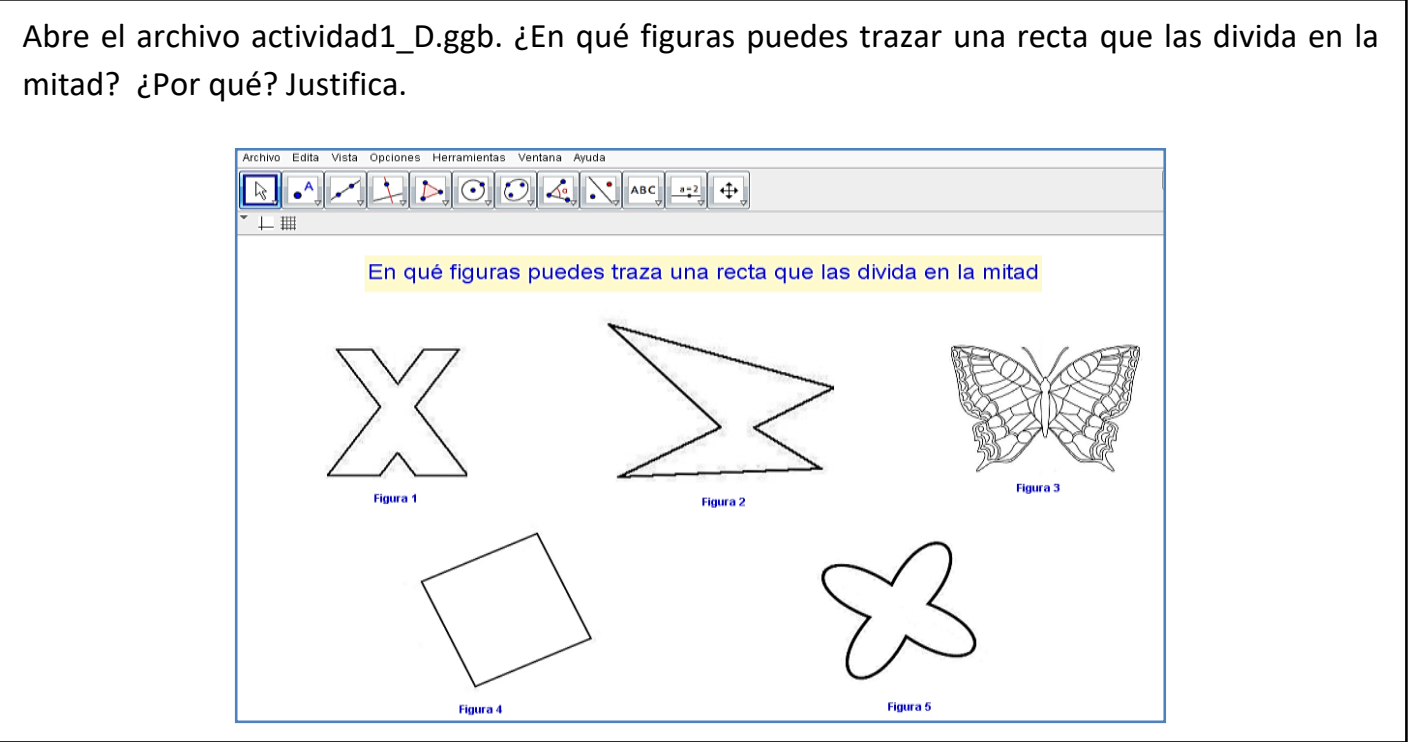

Figura 7. Enunciado de la Actividad 1D

La actividad tuvo como objetivo reconocer y trazar el eje de simetría de cada una de las figuras que esta se pueda trazar. Los estudiantes, deben elegir dos pares de puntos de una de las figuras de tal manera que sean simétrico y trazar su punto medio, ya obtenidos dos puntos medios trazar la recta que pasa por ellos. Este proceso se repetirá en cada figura. Los posibles esquemas de uso son punto medio y recta.

A continuación, mostramos las gráficas donde se muestran los posibles ejes de simetría que los estudiantes deben trazar en el desarrollo de esta actividad, como se puede observar la figura 2 de la actividad no tiene eje debido a que no es simétrica.

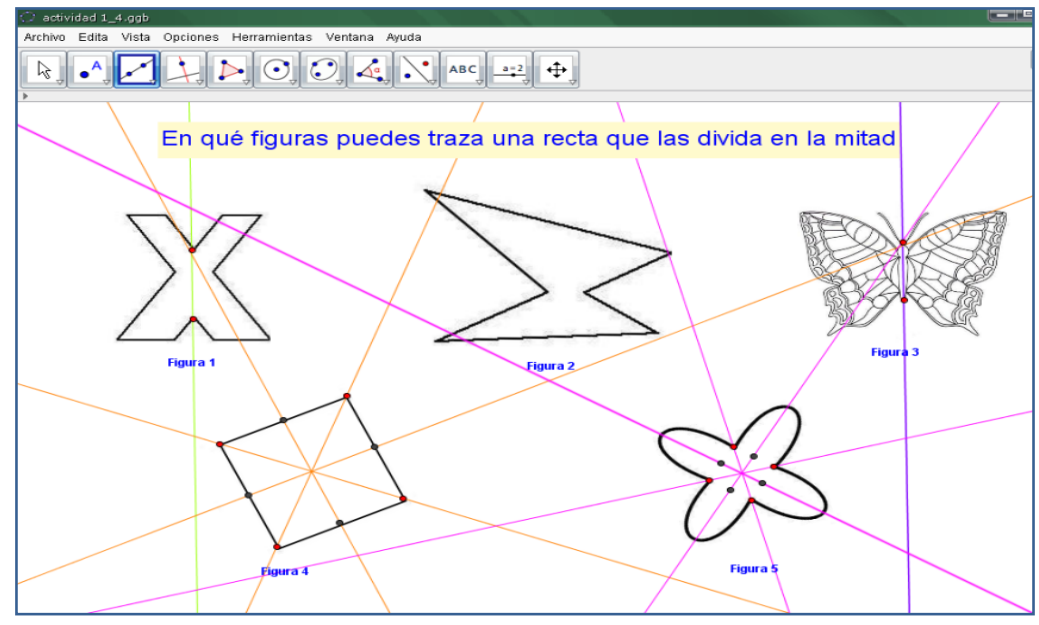

Figura 8. Trazo de los ejes en las figuras simétricas, en cada figura se usó un color distinto.

A posteriori, Marcía traza rectas utilizando la herramienta recta que pasa por dos puntos en las figuras 1; 3; 4 : 5 como se muestra en la figura 9 (a). Logró reconocer que en 8Tangram - Revista de Educação Matemática, Dourados - MS - v.2 n. 3, pp. 28-48 (2019) 
la figura 2 no se puede trazar una recta que la divida en su mitad, en comparación con las rectas trazadas en las otras figuras de la actividad 1D. En la figura 9 (b) se muestra las justificaciones del por qué trazó la recta en las figuras mencionadas anteriormente.

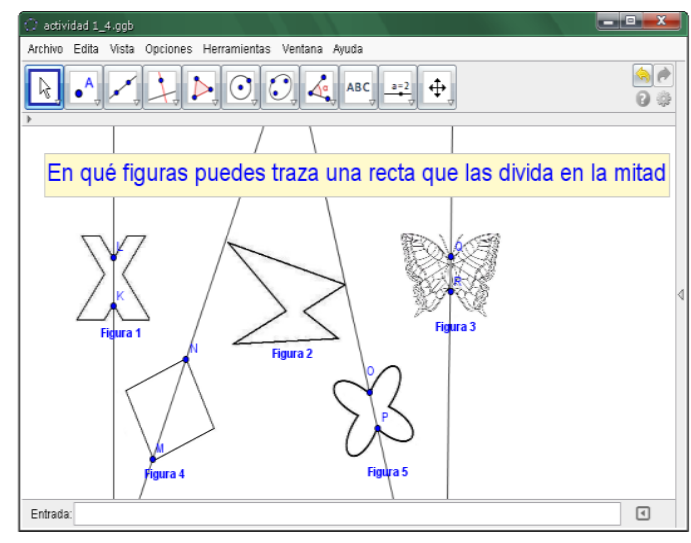

(a)

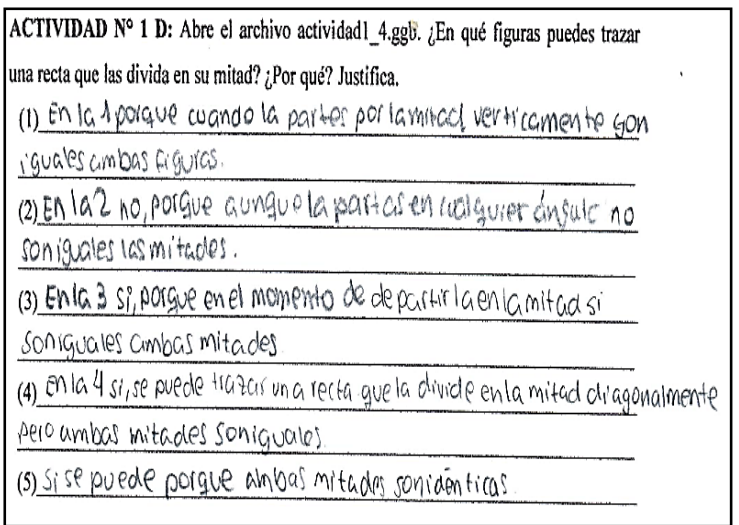

(b)

Figura 9. Trazo de rectas realizado por Marcia en la actividad 1D

De la figura anterior, se pude evidenciar que Marcía ha tratado de trazar la recta varias veces en la figura 2 sin lograr dividirla en su mitad. También creemos que la estudiante ha desarrollado posibles esquemas de uso como congruencia, forma y medida, en especial cuando dice "ambas mitades son idénticas". Marcia logró con el objetivo previsto en nuestro análisis a priori para esta actividad, pero con un procedimiento más de exploración.

A partir del desarrollo de las actividades del tipo 1, Marcia llegó a las siguientes conclusiones (figura 10): Las medidas de los ángulos internos no varían, la distancia de un punto de la figura con su simétrico respecto al eje de simetría siempre es la misma, la forma y el tamaño de una figura no varían en su simétrico, y los segmentos que unen los puntos de la figura con sus simétricos siempre son perpendiculares al eje de simetría.

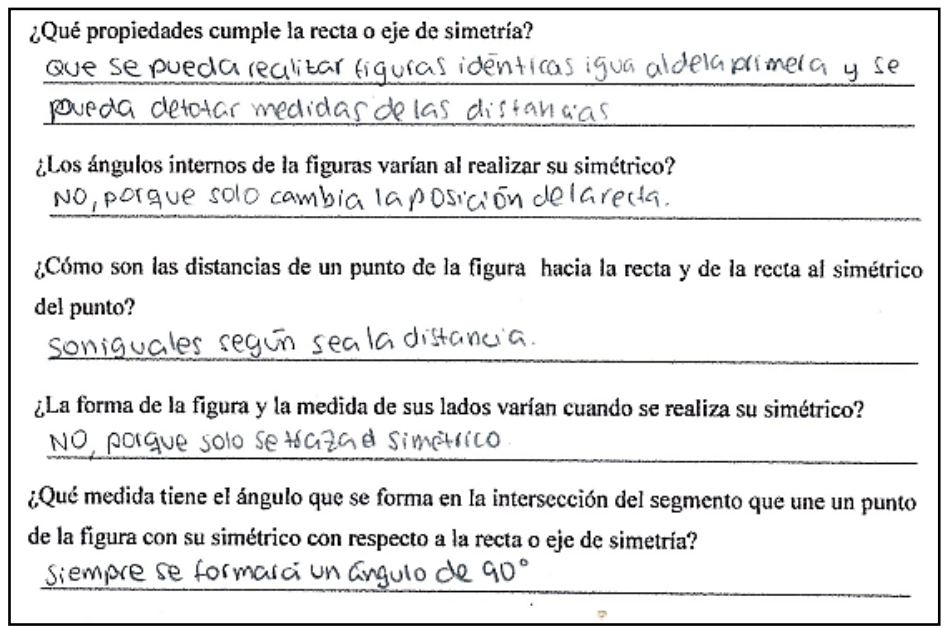

Figura 10. Conclusiones de Marcia en la actividad 1

8Tangram - Revista de Educação Matemática, Dourados - MS - v.2 n. 3, pp. 28-48 (2019) 
Por las conclusiones a las que llegó Marcia y las acciones realizadas en las demás actividades podemos decir que la estudiante presenta indicios de una Instrumentación de las propiedades y del eje de simetría, es decir, se ha generado esquemas de uso y de acción instrumentada de noción de simetría axial.

\section{Actividad 2 A}

El arte textil incaico es una de las más antiguas tradiciones textiles de los Andes y llegó a ser una de las más desarrolladas durante el apogeo inca, debido al uso de diferentes materiales y técnicas (resultado de su cultura híbrida adquirida gracias a las conquistas de otras etnias andinas). Los incas lograron producir una gran variedad de prendas de vestir y elementos de uso cotidiano. Los textiles incas se caracterizan por sus diseños geométricos o tocapus y por la fineza de su técnica. Los incas destacaron por sus tapices y sus mantos de plumas, también de diseños geométricos. Tuvieron un extraordinario sentido de la simetría, reflejado en la repetición de figuras estilizadas dispuestas de una manera sumamente ordenada.
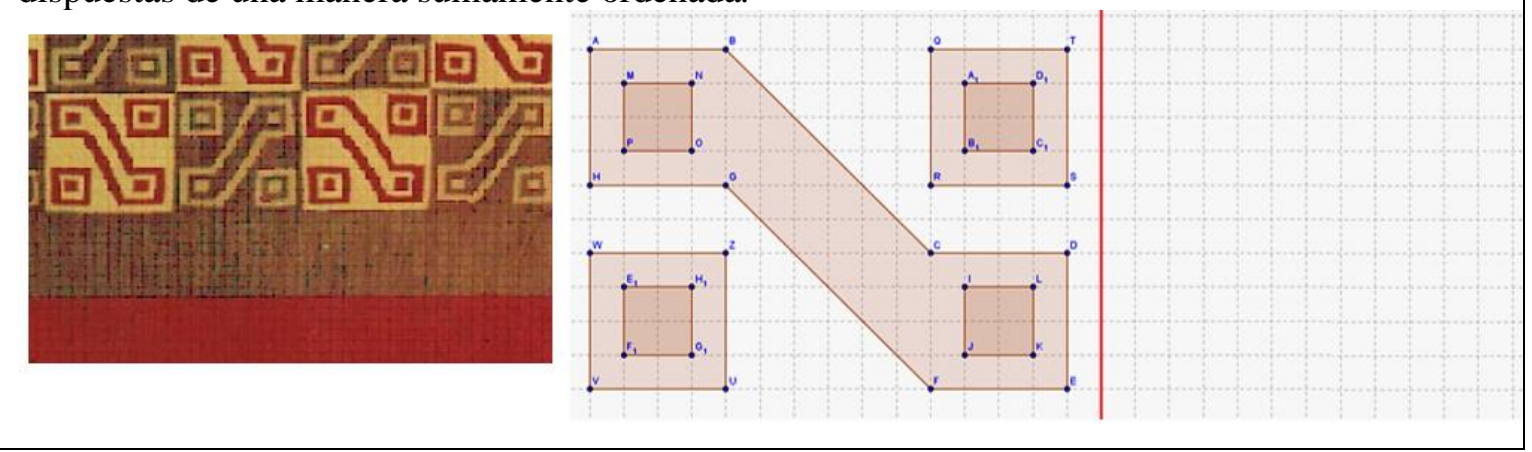

Figura 11. Enunciado de la Actividad 2A

A priori, esta actividad tuvo como objetivo realizar el simétrico de un diseño y verificar que la figura y su simétrico son equidistantes. Los estudiantes deben realizar el simétrico de cada punto de la figura manteniendo la equidistancia al eje de simetría. Luego trazar el simétrico del diseño con el uso de una regla. Esta actividad esta propuesta para realizarse con lápiz y papel, y contiene cuadrículas para que los estudiantes puedan mantener la equidistancia de cada uno de los puntos del diseño y su simétrico con respecto al eje de simetría. Los posibles esquemas de uso son simetría axial, eje de simetría, medida de segmentos, equidistancia. 


\section{Estudio de la génesis instrumental del artefacto simbólico simetría axial}

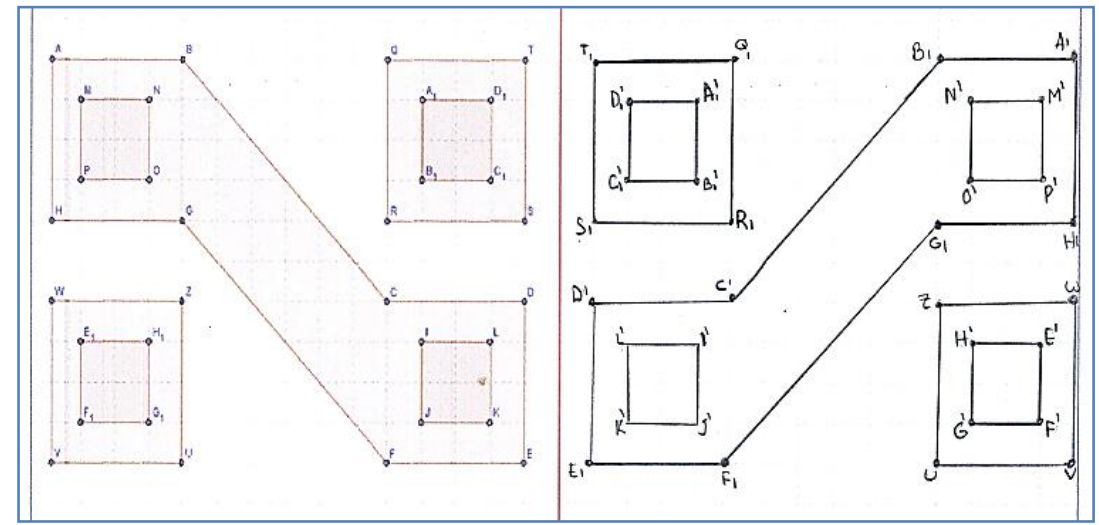

Figura 12. Grafico realizado por Marcia en la actividad 2A

A posteriori, Marcía logra realizar el simétrico del diseño dado como muestra la figura 12. Respetó la orientación del simétrico y ubicó correctamente cada uno sus puntos simétricos. Esto nos da indicios que Marcia esta instrumentada con la noción de simetría ya que lo aplica en situaciones dadas.

\section{Actividad 2 C}

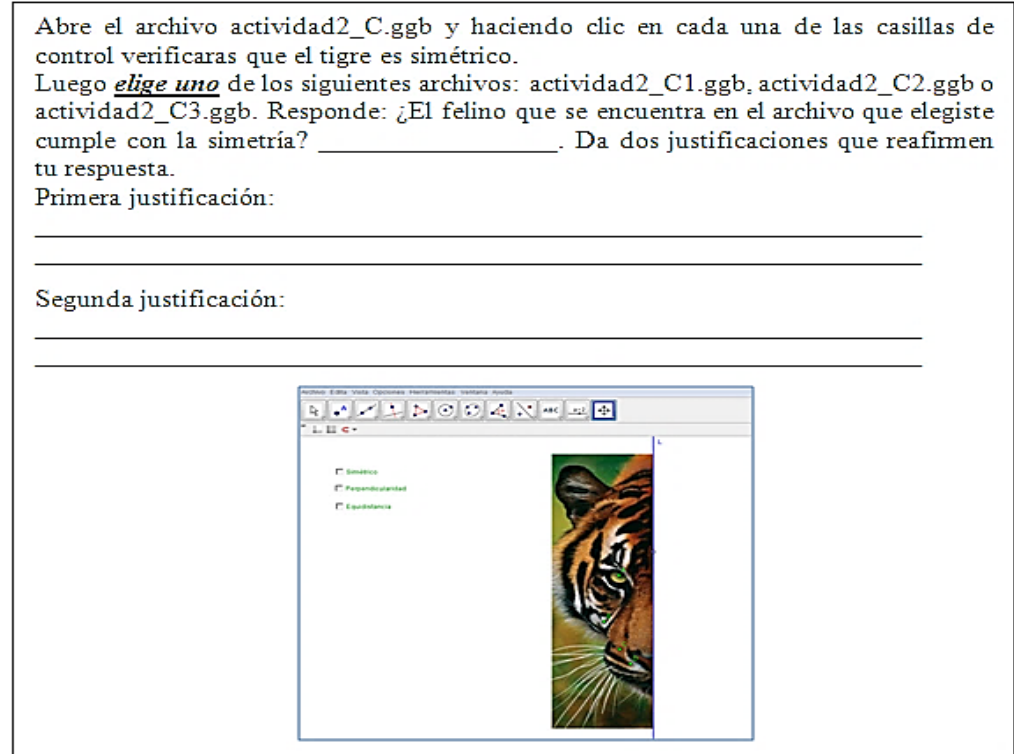

Figura 13. Enunciado de la Actividad 2C.

Primero se les presentó a los estudiantes un archivo de GeoGebra llamado Actividad2_C.ggb, donde se muestra la simetría en la imagen de un felino. Conforme iban haciendo clic en cada una de las tres casillas de control (se puede observar en el link: https://www.geogebra.org/m/k3rqcerq) observaron que se cumple las propiedades de la simetría. Luego, tenían que elegir entre tres archivos denominados Actividad2_C1.ggb, 


\section{Estudio de la génesis instrumental del artefacto simbólico simetría axial}

Actividad2_C2.ggb o Actividad2_C3.ggb. En los cuales tenían que justificar con propiedades de la simetría, si la imagen que se encontraba en el archivo elegido por ellos era simétrica o no.

A priori, la actividad tiene como objetivo reconocer las propiedades de la simetría axial. Pensamos que los estudiantes pueden hacer clic en cada una de las casillas de control, para verificar las propiedades de la simetría y determinar si el tigre es simétrico. Luego de elegir un archivo de los siguientes actividad2_C1.ggb, actividad2_C2.ggb o actividad2_C3.ggb, deben de determinar si el felino es simétrico o no, y justificar haciendo uso de las propiedades. Los posibles esquemas de uso de los alumnos pueden movilizar la noción de eje de simetría, equidistancia, perpendicularidad.

En la figura 14, podemos observar que los dos felinos que se encuentran en los archivos actividad2_C1.ggb y actividad2_C2.ggb, no son simétricos porque no cumple con la propiedad de la equidistancia, la perpendicularidad del eje de simetría con el segmento que une un punto de la figura inicial con su simétrico. Por el contrario, el felino que se encuentra en el archivo actividad2_C3.ggb, sí cumple con las propiedades y por ende es simétrico. En el archivo actividad2_C1.ggb, la imagen del felino no muestra simetría porque no cumple la perpendicularidad entre el que podría ser el eje de simetría y el segmento que se forma entre uno de los puntos del lado izquierdo con quien vendría a ser su simétrico en el lado derecho. Tampoco cumple la equidistancia y, además, en el lado izquierdo el felino presenta una pata que no se encuentra en lado derecho. En el archivo actividad2_C2.ggb, la imagen del felino no muestra simetría porque no cumple la perpendicularidad entre el que podría ser el eje de simetría y el segmento que se forma entre uno de los puntos del lado izquierdo con quien vendría a ser su simétrico en el lado derecho. Además, no cumple la equidistancia de los puntos con respecto a la recta y la orientación con respecto al eje no varía. En el archivo actividad2_C3.ggb, la imagen del felino sí cumple con la perpendicularidad, la equidistancia respecto al eje de simetría y el cambio de orientación respecto al eje, por tanto, si presenta simetría.

8Tangram - Revista de Educação Matemática, Dourados - MS - v.2 n. 3, pp. 28-48 (2019) 


\section{Estudio de la génesis instrumental del artefacto simbólico simetría axial}

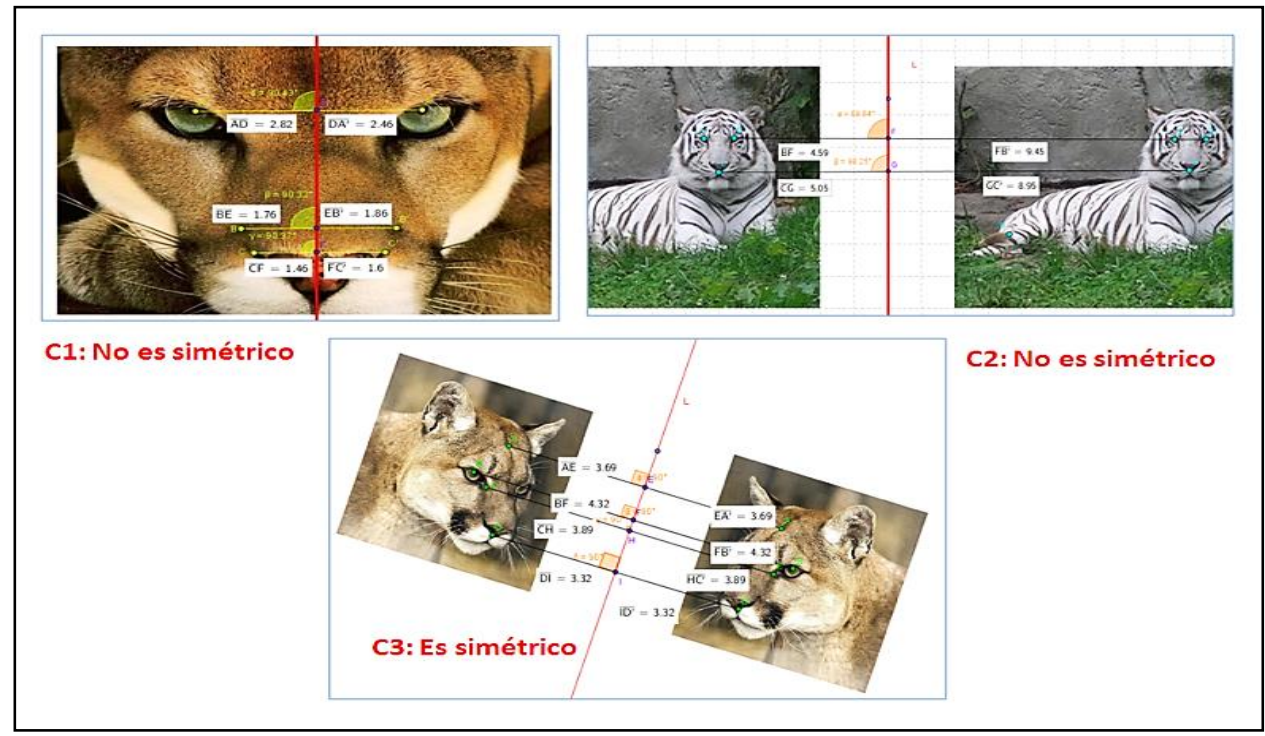

Figura 14. Solución de la actividad 2C

A posteriori, Marcia realizó las siguientes acciones: Escogió el archivo C1, midió ángulos, trazó segmentos, midió segmentos y trazó puntos de intersección entre los segmentos y la recta. Como se pude observar en la figura 15 (a). Marcia logró reconocer que el Felino que se encuentra en el archivo C1 no cumplía con la simetría. Para determinarlo ella hizo medida de ángulos, segmentos, trazó puntos de intersección que le permitieron realizar estos procedimientos. La estudiante, manifestó, figura 15 (b), que no se cumplía que los ángulos formados entre la intersección de los segmentos que trazó y el supuesto eje de simetría midieran $90^{\circ}$. Tambíen que la medida de los puntos de la figura no eran equidistante con el eje de simetría.

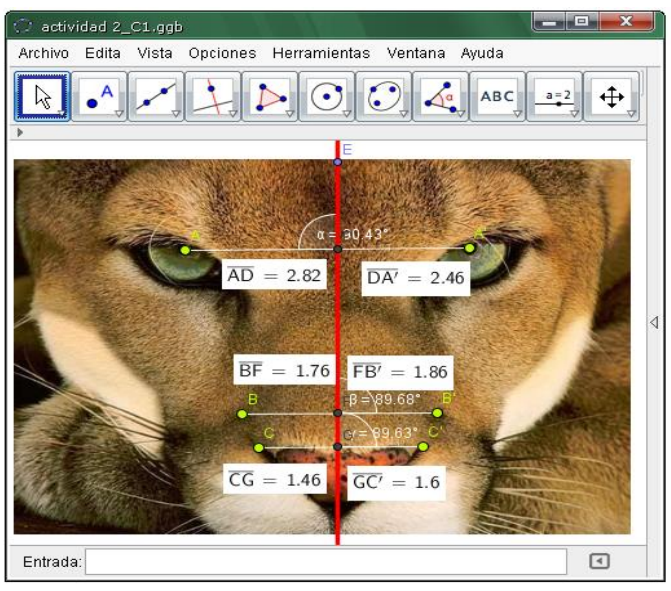

(a)

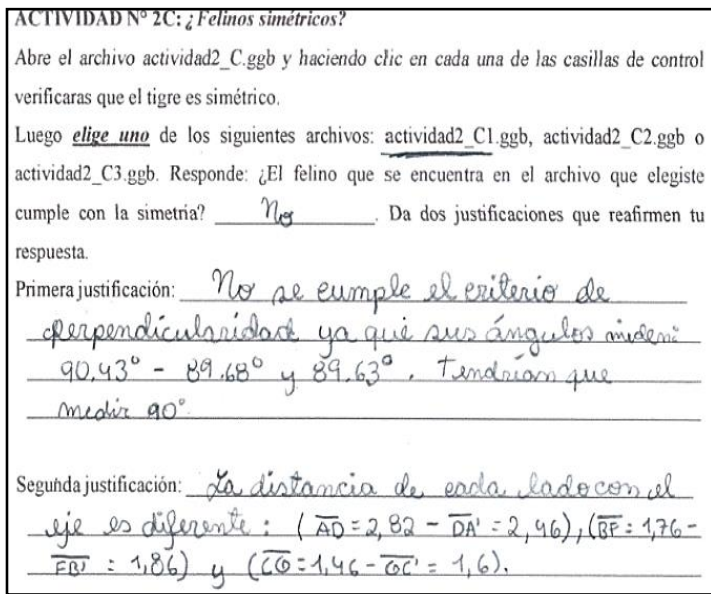

(b)

Figura 15. Procedimiento y respuestas de Marcia en la actividad 2C.

8Tangram - Revista de Educação Matemática, Dourados - MS - v.2 n. 3, pp. 28-48 (2019) 


\section{Estudio de la génesis instrumental del artefacto simbólico simetría axial}

Por sus acciones, antes mencionadas, y por la movilización de conceptos como eje de simetría, equidistancia y perpendicularidad, usadas para el desarrollo de esta actividad, podemos darnos cuenta que Marcia ha logrado Génesis Instrumental de la noción de simetría axial y que puede aplicar sus propiedades.

\section{CONSIDERACIONES FINALES}

Mediante el análisis de las acciones de la estudiante, Marcia, hemos podido identificar los posibles esquemas de uso y de acción instrumentada, pudiendo evidenciar que el proceso de la Génesis instrumental del artefacto simbólico Simetría Axial se dio en la estudiante, lo cual se ratificó en su solución de la actividad 2 C.

Luego de nuestra experimentación, podemos decir, que la estudiante instrumentaliza e instrumenta la noción de simetría (Génesis Instrumental), ya que por medio de sus acciones pudimos observar cómo aprendió esta noción mediante actividades planteadas y mediante sus conclusiones de las actividades $1 \mathrm{C}, 1 \mathrm{D}, 2 \mathrm{~A}$ y $2 \mathrm{C}$ que escribió en su ficha de trabajo. Estas pusieron de manifiesto, las conjeturas a las cuales llegó, reconociendo propiedades de este objeto matemático.

Es importante destacar que, en la Génesis instrumental, la estudiante maneja desde ya un repertorio de esquemas y que estos son acomodados o generalizados al nuevo artefacto para atribuirle un cambio de significado. Por ejemplo, en nuestra investigación, los esquemas como mediatriz, punto medio, perpendicularidad y equidistancia son parte del repertorio que moviliza la estudiante para atribuirle un significado a la simetría axial.

El trabajar con tecnologías (GeoGebra, lápiz y papel), y el saber de la existencia de un enfoque de estudio en educación matemática con las tecnologías, Enfoque Instrumental de Rabardel (1995), que nos da una perspectiva que por medio de las acciones de los estudiantes podamos tener indicios de la movilización o creación de esquemas mediados por el uso de las tecnologías.

\section{AGRADECIMENTOS}

El presente artículo ha sido posible gracias al apoyo de la Maestría en Enseñanza de las Matemáticas-Escuela de Posgrado de la Pontificia Universidad Católica del Perú, especialmente, al grupo de investigación Tecnologías y Visualización en Educación Matemática TecVEM, de la Maestría en Enseñanza de las Matemáticas y del IREM/PUCP.

8Tangram - Revista de Educação Matemática, Dourados - MS - v.2 n. 3, pp. 28-48 (2019) 


\section{REFERENCIAS BIBLIOGRÁFICAS}

Almouloud, S.A. (2007). Fundamentos da didática da matemática. Curitiba: Ed. UFPR.

Artigue, M. (1995). Ingeniería didáctica. En Artigue, M., Douady, R, Moreno, L., Gómez, P. (Eds.). Ingeniería didáctica en educación matemática: un esquema para la investigación y la innovación en la enseñanza y aprendizaje de las matemáticas. Bogotá: Grupo editorial Iberoamérica

Bellemain, F. B., \& Trouche, L. (2016). Compreender o trabalho do professor com os recursos de seu ensino, um questionamento didático e informático. I Simpósio LatinoAmericano de Didática da Matemática, Nov 2016. Bonito, Brasil. Recuperado de: https://hal.archives-ouvertes.fr/hal-01560233

Bettini, G. (2017). Un'orchestra di strumenti matematici. Macchine matematiche, software di geometría dinamica e LIM nella scuola media1. Rivista Didattica della matematica. Dalla ricerca alle pratiche d'aula. V4, p. 65 - 92. Recuperado de: http://www.rivistaddm.ch/index.php/2017-01-volume/2017-01-bettini/

Denzin, N. y Lincoln, Y. (1994). Introduction entering the field of qualitative research, en Denzin, N. y Lincoln, Y (eds). Handbook of qualitative research. Thousand Oaks, California.

Ferreira, Paula (2005). Isometrias: Análise de documentos curriculares e uma proposta de situações de aprendizagem para o ensino médio. (Tesis de maestria). Pontificia Universidade Católica de São Paulo, Brasil.

Gallegos, D y Peña, A. (2012). Las TIC en geometría, una nueva forma de enseñar. Bogotá: Ediciones de la U.

García-Cuéllar, D. (2014). Simetría axial mediada por el GeoGebra: un estudio con estudiantes de primer grado de educación secundaria. (Tesis de maestría). Pontificia Universidad Católica del Perú, Perú. Recuperado de: 10.13140/RG.2.2.13450.47048

Jaime, A (1993). Aportes a la interpretación y aplicación del modelo Van Hiele: la enseñanza de las isometrías del plano. La evaluación del nivel de razonamiento. (Tesis doctoral). Universidad de Valencia, España.

Ledermand, L. (1996). La partícula divina: Si el universo es la respuesta, ¿cuál es la pregunta? Barcelona: Grijalbo Mondadori, S.A.

Lima, I. (2006). De la modélisation de connaissances des élèves aux décisions didactiques des professeurs: étude didactique dans le cas de la symétrie orthogonale. Thèse 'Université, Université Joseph Fourier, Grenoble.

Perú, Ministerio de Educación (2005). Unidad de Medición de la Calidad. Evaluación Nacional del Rendimiento Estudiantil 2004. Informe pedagógico de resultados. Formación matemática: Tercer grado y Quinto de secundaria. Recuperado de http://www2.minedu.gob.pe/.

8Tangram - Revista de Educação Matemática, Dourados - MS - v.2 n. 3, pp. 28-48 (2019) 
Perú, Ministerio de Educación del Perú (2011). Orientaciones para el trabajo pedagógico 2011 del área de matemática. Recuperado de: goo.gl/AHwUTD

Perú, Ministerio de Educación del Perú (2016). Resultados de la evaluación censal de estudiantes 2016. Lima. Recuperado de: http://umc.minedu.gob.pe/resultadosece2016/

Rabardel, P. (1995). Les hommes et les technologies: aproche cognitive des instrumentns contemporains. Paris: Armand colin.

Rabardel, P. (2011). Los hombres y las tecnologías: Visión cognitiva de los instrumentos contemporáneos. (Trad. por M. Acosta) Colombia: Universidad Industrial de Santander.

Santillana. (2018), Matemática 1. Editorial Santillana: Perú.

Salazar, J.V.F. (2009). Gênese instrumental na interação com Cabri 3D: um estudo de transformações geométricas no espaço. (Teses). Pontificia Universidade Católica de São Paulo, Brasil.

Trouche, L. (2004). Managing the complexity of human/machine interactions in computerized learning environments: guiding students' command process through instrumental orchestrations. International Journal of Computers for Mathematical Learning 9: 281-307.

Ulian, C. (2008). Possibilidades da aprendizagem de transformações geométricas com o uso do Cabri-Geomètre. Recuperado de http://www.pucsp.br/pos/edmat

Vergnaud, G. (1996). A teoria dos campos conceptuais. En Jean Brun (org), Didáctica das matemáticas. (pp. 155-189). Lisboa: Horizontes pedagógicos.

Enviado:27/11/2018 Aceito:06/04/2019 